\title{
Analysis of the Pantograph Aerodynamic Loads under Crosswind
}

\author{
Yanzhe Han, Qingquan Gao, Jikun Ma, Meng Zhao and Yanhong Xi \\ School of Civil Engineering and Architecture, Beijing Jiaotong University, Beijing 100044, China \\ *Corresponding author
}

\begin{abstract}
The pressure correction method based on N-S equation, using the split SIMPLE algorithm, the crosswind conditions of pantograph's aerodynamic characteristics numerically simulation analyzed. The results show that with the wind direction increasing, the pantograph's aerodynamic load is nonlinear, the lift coefficient increases with the increase of wind angle; at the same time as the wind speed increases, the slope of lift coefficient increases; pantograph overturning moment, cornering coefficient increase as wind direction angle increases. The lateral force coefficient increases monotonically with wind direction angle increases; to flow around the contact wire and slide, slide bracket, forming a large area in the rear of the slide wake zone near the leeward side, small scale in the slide and contact line vortex accounted for the major part, while the two slide end and separate the vortex interaction support And the results can provide a basis for improving stability and safety of the pantograph of the crosswind.
\end{abstract}

Keywords-high speed train; pantograph; crosswind; flow field; aerodynamic load

\section{INTRODUCTION}

The pantograph when running in high speed operation, due to horizontal wind's effect, the aerodynamic drag, lift force, lateral force will have significant changes, which has affected the pantograph's operation stability and flow quality caused by unfavorable effects, so we need to analyze the dynamic load of pantograph under crosswind systematically. The pantograph's good aerodynamic performance is the important factors to ensure that the following performance of pantograph catenary system, stability and reduce pantograph wear and reduce aerodynamic drag, as well as the aerodynamic noise. The safety of the pantograph is in the influence of many factors, the crosswind is a very important one of them. Therefore, under the condition of wind the study on the dynamic characteristics of electric load of arch air is particularly important and urgent. In terms of the electric performance of the pantograph gas, many scholars have done a lot of numerical simulations and analyzes the change rule [1-4].But the study under the cross wind bow net literature is less, in this respect there is no systemic study. According to the actual characteristics of the project, the author analyzed the aerodynamic load characteristics of the pantograph in the crosswind using numerical simulation methods, in order to provide the foundations of theoretical research to further improve the dynamic performance of the pantograph catenary and improve the aerodynamic noise simulation.

\section{AERODYNAMiC LOAD CALCULATION MODEL OF PANTOGRAPH}

\section{A. Simplified Pantograph Model}

This article calculated, using simplified model of the pantograph, and the geometric shape of pantograph slide, upper arm and lower arm lever remains unchanged, and the bow Angle is constant. Simplifying the stent and chassis parts, geometry shape of pantograph is shown in figure 1 .

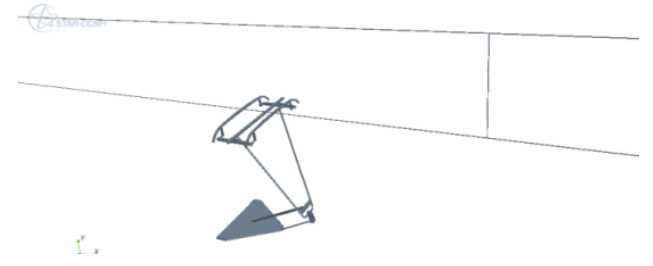

FIGURE I. PANTOGRAPH GEOMETRY MODEL

\section{B. Computational Domain and Grid Partition}

The pantograph above the computational domain size is more than 10 times the height of the pantograph, entrance and the mainstream wind, wake zone set up enough length, experience is the size of the computational domain can basically ignore the influence of the pantograph around flow field, as shown in Figure 2.

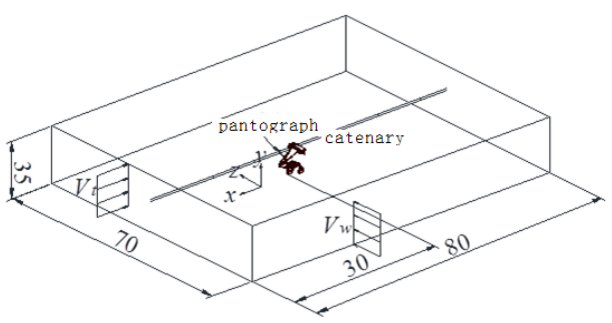

FIGURE II. SET OF CALCULATION FIELDS

The computational domain grid with Trim grid, in an arch and the surface of the body wall function method for processing [5]. in order to ensure the smooth connection with the mainstream area boundary, setting the boundary grid thickness is $6.8 \mathrm{~mm}$, the boundary layer mesh is divided into 6 layers, the first layer near the wall of the grid from the wall distance is $0.2 \mathrm{~mm}$. And by regional electric variation near arch and contact line field of encryption, as shown in Figure 3. 


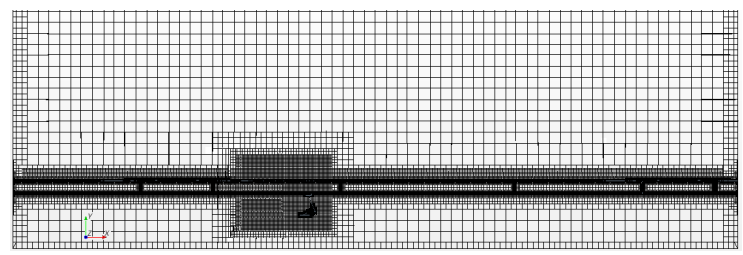

FIGURE III. PARTITION OF MESH

\section{Setting of Calculation Conditions}

The settings of boundary conditions, have an important effect on the calculation results of the numerical model. According to the relative motion criterion, numerical simulation of the boundary conditions are set as follows: in the running direction of the train, the boundary conditions of the entrance is the entrance speed , and speed value is $97.22 \mathrm{~m} / \mathrm{s}$, the boundary conditions of the export is export pressure, relative to the atmospheric pressure is 0 ; in the horizontal direction. The boundary conditions for the entrance for entrance speed, outlet boundary conditions for pressure outlet, relative to the atmospheric pressure is 0 ; the gas boundary is the slip boundary. The floor is no slip boundary and setting speed, its value is $97.22 \mathrm{~m} / \mathrm{s}$, near the entrance to a small range of slip boundary.

The train at $350 \mathrm{~km} / \mathrm{h}$, run under different wind speed and wind direction,air flow around the surface of the pantograph is relatively complex, forming a three-dimensional flow field and heat transfer process are subjected to the most basic law of physics domination, at the same time acts as the basis of the setting of boundary conditions. The momentum equation, and continuity equation simultaneous solution of the flow field;Solving boundary layer by Low Reynolds number $k-w$ equation, the general form of flow control equations are as follows [6-8]:

$$
\begin{aligned}
& \frac{\partial(\rho \varphi)}{\partial t}+\operatorname{div}(\rho \varphi \mathbf{U})=\operatorname{div}\left(\Gamma_{\varphi} \operatorname{grad} \varphi\right)+S_{\varphi} \\
& \rho-\text { density of fluid;t-time; U-fluid velocity }
\end{aligned}
$$

vector; $\varphi$-generic variable $\left(u, \mathrm{v}, w, \mathrm{~T}, m_{l}\right) ; S_{\varphi}-$ generalized source term; $\Gamma_{\varphi}$ —generalized diffusion coefficient

Because of the complexity of the turbulent flow, get the direct solution of the equations is difficult, so using the method of calculating the time averaged equations and turbulence model, namely the turbulent flow is regarded as time average flow and flow pulsation of the stack. At this point we need to solve the equation about turbulence model.In this paper we use SST $k-w$ model.It combines the advantages of the prototype of $k-w$ and the model of $k-\varepsilon$, The equation is as follows:

$$
\begin{aligned}
& \frac{D \rho \omega}{D t}=\frac{\gamma}{v_{t}} \tau_{i j} \frac{\partial \bar{u}_{i}}{\partial x_{j}}-\beta \rho \omega^{2} \\
& +\frac{\partial}{\partial x_{j}}\left[\left(\mu+\sigma_{\omega} \mu\right) \frac{\partial \omega}{\partial x_{j}}\right]+2\left(1-F_{1}\right) \rho \sigma_{\omega 2} \frac{1}{\omega} \frac{\partial k}{\partial x_{j}} \frac{\partial \omega}{\partial x_{j}}
\end{aligned}
$$

$k$-turbulence kinetic energy; $\omega$-vorticity fluctuation intensity

\section{NUMERICAL SIMULATION RESULTS ANALYSIS}

\section{A. Calculation Conditions}

Calculation of train running speed is $97.22 \mathrm{~m} / \mathrm{s}$, wind speed of $15,20,25,30 \mathrm{~m} / \mathrm{s}$, the wind angle of 0 degrees to 90 degrees, 10 degree intervals of a condition calculation, analysis of pantograph drag, lift, lateral force variation.

\section{B. Calculation Results Analysis}

The pantograph is a complex system composed of a slide plate, an upper frame, a lower arm rod, etc., and the flow direction and the flow direction of each component are different, so the aerodynamic load distribution is more complicated.

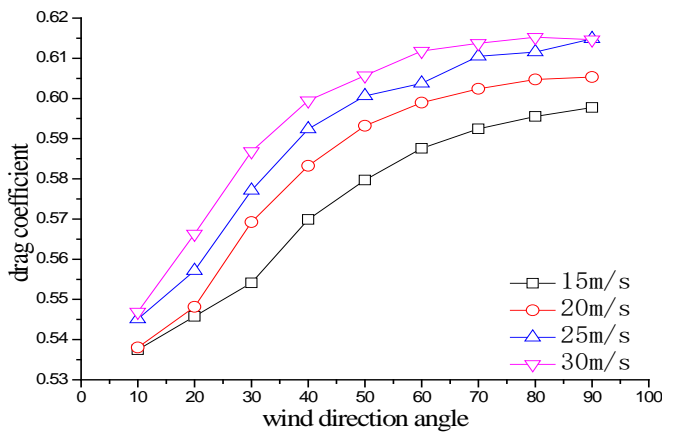

FIGURE IV. VARIATION CURVE OF DRAG COEFFICIENT

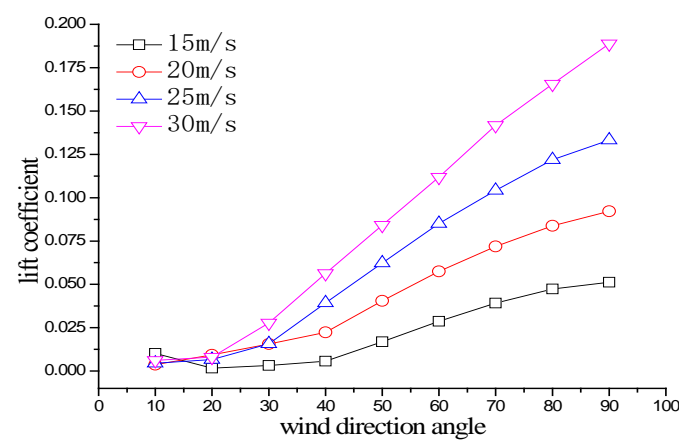

FIGURE V. VARIATION CURVE OF LIFT COEFFICIENT

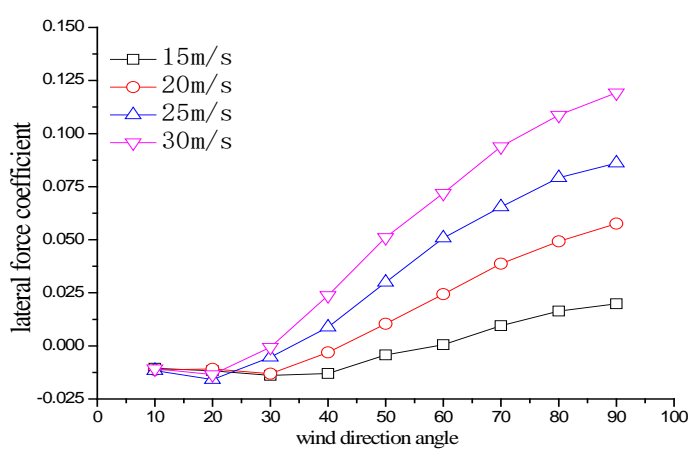

FIGURE VI. VARIATION CURVE OF LATERAL FORCE COEFFICIENT

The pantograph drag coefficient of the wind direction angle increases, and the maximum value appeared in the wind speed $30 \mathrm{~m} / \mathrm{s}$ conditions; with wind speed for the $30 \mathrm{~m} / \mathrm{s}$ operating mode as an example, with the increase of wind direction 
angle,drag coefficient increased by 12.4\%. From Figure 4 and 5, they show the pantograph lift coefficient increase with the increase of wind direction angle. At the same time as the wind speed increases, the slope of lift coefficient curve increases. We can see from Figure 6 the pantograph lateral force coefficient increase as the wind direction angle increases, consistent with its variation with the lift coefficient, monotonically increasing, the maximum lateral force coefficient appeared in the working condition of horizontal packing winds of up to $30 \mathrm{~m} / \mathrm{s}$ wind.Train running speed is constant,the pantograph aerodynamic load coefficient of the most unfavorable conditions appear in the working condition of horizontalmaximum wind speed, wind direction angle of the largest, and with the increase of wind speed and wind direction angle, the influence of the pantograph aerodynamic characteristics is more significant, in the strong wind environment, the focus should be on the wind the effect of the horizontal wind of the pantograph.

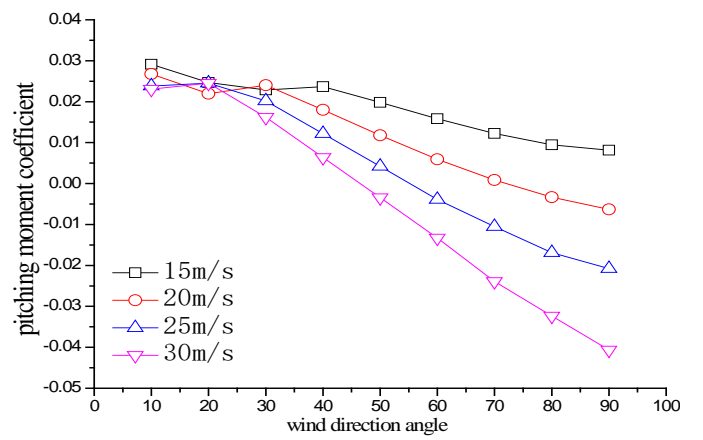

FIGURE VII. PITCHING MOMENT COEFFICIENT CURVE

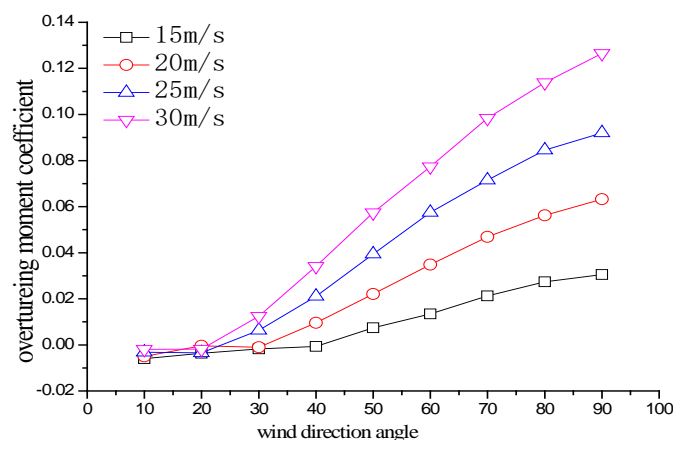

FIGURE VIII. OVERTURNING MOMENT COEFFICIENT CURVE

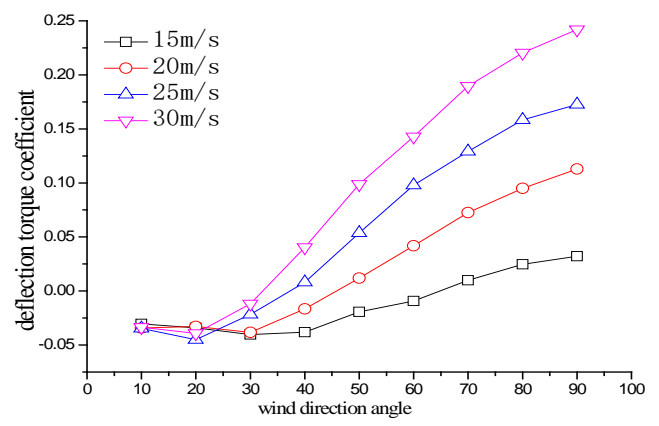

FIGURE IX. DEFLECTION TORQUE COEFFICIENT CURVE
Figure 7 to 9 respectively for pantograph pitching moment, overturning moment and yawing moment vary with the chance of wind direction angle of the curve, we can see from Figure 7, the pantograph pitching moment coefficient decreases as the wind direction angle increase, except for $15 \mathrm{~m} / \mathrm{s}$ wind speed conditions, pitching moment coefficient are of negative value (in contrast with the specified direction); with the increase of wind speed, the slope of the curve of pitching moment increases by 2 times. Figure 8 and Figure 9 show that the pantograph overturning moment, and cornering coefficient increase as the wind direction angle increase; in the case of horizontal wind speed is larger, overturning moment coefficient and yawing moment coefficient change obviously, in the condition of small wind speed, the change trend is flat.

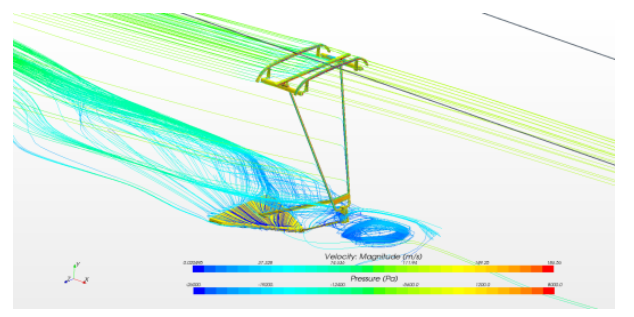

FIGURE X. PANTOGRAPH STREAMLINE AND SURFACE PRESSURE

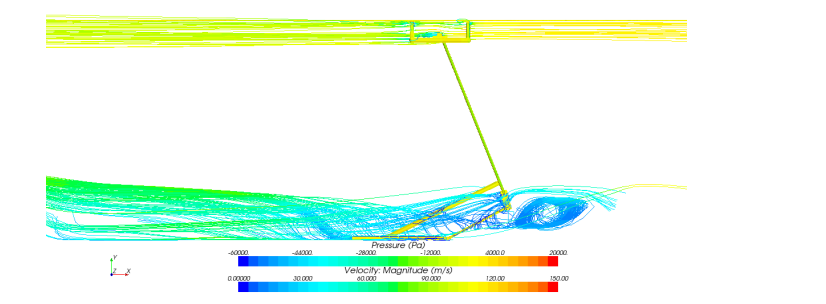

FIGURE XI. STREAMLINE DISTRIBUTION OF CROSS SECTION

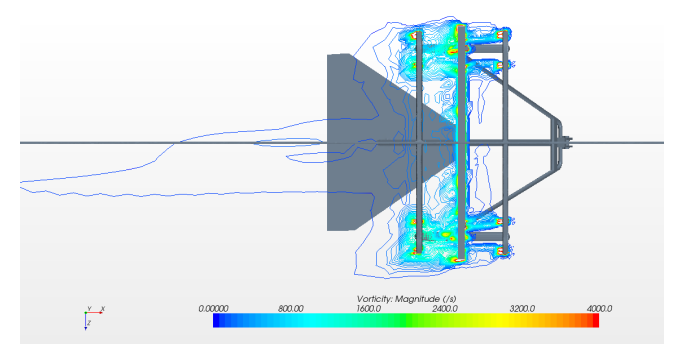

FIGURE XII. DISTRIBUTION OF VORTICITY IN LONGITUDINAL SECTION

Figure 10 to 12 show the distribution of the pantograph, the distribution of the surface pressure and the distribution of the cross section of the pantograph. From the figures, the stream flow around the contact wire and slide, slide bracket, forming a large area in the rear of the slide wake zone near the leeward side, small scale on the slide and the contact line the vortex accounted for the major part, while the two end face and the support from the disposition of slide vortex interaction, distribution is very complex. The mutual influence of catenary and slide the flow around mainly manifested in the small scale vortex interaction, the dissipation and shedding, common process area generation of effects of flow slide nearby. In the rear of pantograph exists visible large scale vortexes, the flow 
field characteristics of pantograph under the arm, push rod and the bottom of the upper arm rod significantly influences on the flow field, the large scale vortex separation after spread, the downstream flow around pantograph lower arm rod and other components continue to produce visible after large scale separation vortex, work together to form a broad range of low speed wake zone. In addition to being affected by the rear flow field, the lower to the upper arm flow around push rod and the rod and hinge connection after separation vortex formed by small scale and large scale separation vortex and impact on the arm of the flow field around. The flow field around the middle part of the upper arm rod is affected basically by the coming flow and with the rest of the bar around the interaction of the flow field of the weaker.

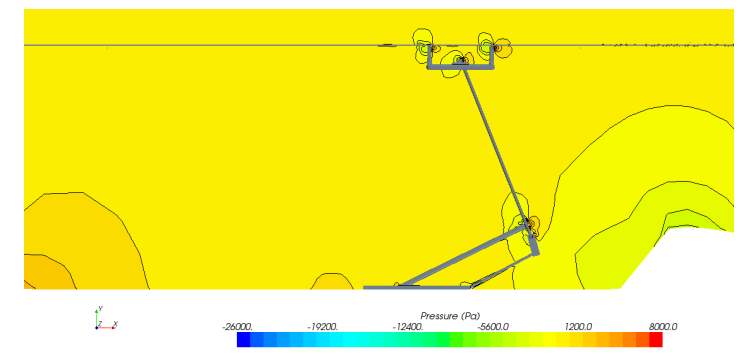

FIGURE XIII. WHEN THE PRESSURE DISTRIBUTION OF WIND SPEED 20M/S

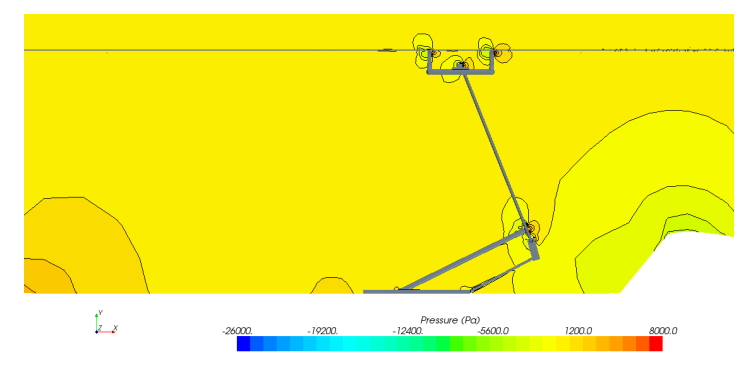

FIGURE XIV. WHEN THE PRESSURE DISTRIBUTION OF WIND SPEED 25M/S

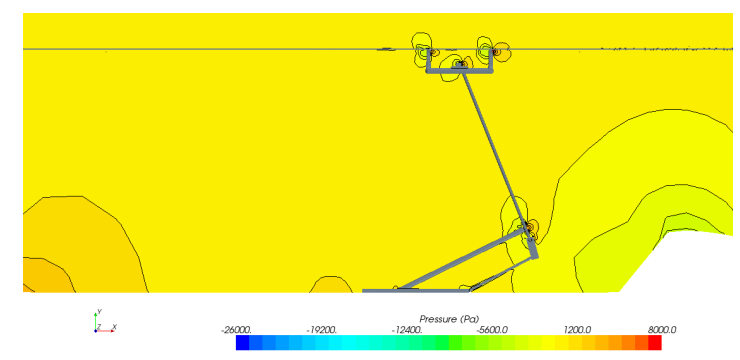

FIGURE XV. WHEN THE PRESSURE DISTRIBUTION OF WIND SPEED $30 \mathrm{M} / \mathrm{S}$

Figure 13 to 15 show the train speed is $350 \mathrm{~km} / \mathrm{h}$, wind direction angle is 30 degrees, crosswind speed is $20 \mathrm{~m} / \mathrm{s}, 25 \mathrm{~m} / \mathrm{s}$ and $30 \mathrm{~m} / \mathrm{s}$ by drawing longitudinal section pressure field distribution. When crosswind speed increases or wind angle increases,the range of low pressure area near the pantograph shroud increases, when the wind direction angle of 30 degrees (yaw speed angle of 3 degrees to 6 degrees), the low pressure area affects the scope of the pressure distribution around the pantograph under the arm, upper arm and the lower end of the rod; when the wind direction angle of 60 degrees (yaw velocity angle of 4 degrees to 12 degrees) the range of low pressure area is further increased;when the crosswind speed of $30 \mathrm{~m} / \mathrm{s}$, from the rear dome to pantograph wake region, forming a large area of low pressure area, at the same time the low pressure area have influence on the contact net. In the slide,the skateboard support and the joint of upper arm and push rod, a small range of low pressure area appears, the area has little change along with the change of horizontal wind speed and wind direction angle. By the effect of the pressure field near the pantograph, the pressure distribution around the end of the dome as well as the junction of the central train and tail vehicle changes, with the increase of crosswind speed and wind direction angle, the area of the high pressure part,which is around the end of dome decreases.

\section{CONCLUSION}

1) As the wind direction angle increases, the change of pantograph aerodynamic load is nonlinear, the pantograph drag coefficient increases as the wind direction angle increases, and the maximum value appeared in the wind speed $30 \mathrm{~m} / \mathrm{s}$ condition; lift coefficient increases while the wind direction angle increases; at the same time as the wind speed increases, the slope the lift coefficient curve increases. The coefficient of lateral force increases monotonically with wind direction angle increasing.

2) The pantograph overturning moment and cornering coefficient increase when the wind direction angle increases; in the case of high wind speed, overturning moment coefficient and yawing moment coefficient change obviously. In the condition of smaller wind speed they change trend.

3) The stream flow around the contact wire and slide, slide bracket, forming a wide range of low speed wake zone, the small scale in the slide and contact line vortex accounted for the major part, while the two end face and the support from the slide disposition vortex interaction, distribution is very complex. Interaction of contact net and slide the flow field around the main performance in the interaction of small scale vortex shedding, the dissipation and the common process of reforming, influencing the flow field near the skateboard.

4) To stream flow around the upper arm at the lower end of the push rod, and the formation of joint after small scale vortex and large scale vortex and then on the next boom. The influence of flow around the upper arm of the basic flow is affected only by the flow and the other member interaction flow field is weak.

5) In the slide, slide and the upper arm bracket rod and a push rod connected at low pressure area in small areas, the area along with the change of wind speed and wind direction are very small. By the effect of electric field near the pressure arch, dome end and central train, at the junction of the tail vehicle pressure distribution changes.

\section{ACKNOWLEDGMENT}

Project supported by College Student Research and CareerCreation Program of Beijing city. 


\section{REFERENCES}

[1] CAI Guohua. Aerodynamic Characteristic Measurements on Pantograph of Super Express train[J]. Experiments and Measurements in Fluid Mechanics, 2004,(18-1):54-56. (in Chinese)

[2] Wu Yan, Wu Junyong, Zheng Jihao. A Study on Dynamic Simulation of High-speed Pantograph[J], 2008 National Doctoral Forum : Electrical Engineering Proceedings, 2008: 909-910. (in Chinese)

[3] ZHOU Dongpeng, WU Jun-yong, WU Yan. Finite element simulation on pantograph - catenary dynamic system for Beij ing-Tianj in intercity high - speed railway[J]. Journal of Traffic and Transportation Engineering, 2009,(9-1): 26-28.(in Chinese)

[4] Ben Diedrichs. Aerodynamic Calculations of Crosswind Stability of a High-speed Train Using Control Volumes of Arbitrary Polyhedral Shape[J]. BBAA VI International Colloquium on: Bluff Bodies Aerodynamics \& Applications, 2008(20-24):4-6.

[5] WANG Fujun. Computational Fluid Dynamics Analysis [M]. Tsinghua University Press,2005:131-132.(in Chinese)

[6] Samuel Holmes, Elton Toma, Martin Schroeder. High-Speed Passenger and Intercity Traun Aerodynamic Computer Modeling[J]. The 2000 International Mechanical Engineering Congress \& Exposition. 2000, (510):1-7.

[7] TAO Wenquan. Numerical Heat Transfer[M]. Second Edition. Xi'an. Xi'an Jiao Tong University Press.2001:1-18.(in Chinese)

[8] WU Qinghai, ZHOU Hongwei, ZHU Yonggeng. High-speed train Numerical Simulation of Turbulent Flow Field Calculation [J]. Journal of the China Railway Society, 2002,(3-24):102-103. (in Chinese) 